\title{
Influence of spatial heterogeneity of local surface albedo on the area-averaged surface albedo retrieved from airborne irradiance measurements
}

\author{
E. Jäkel ${ }^{1}$, M. Wendisch ${ }^{1}$, and B. Mayer ${ }^{2,3}$ \\ ${ }^{1}$ Leipzig Institute for Meteorology (LIM), University of Leipzig, Leipzig, Germany \\ ${ }^{2}$ Meteorological Institute, Ludwig-Maximilians-University, Munich, Germany \\ ${ }^{3}$ Deutsches Zentrum für Luft- und Raumfahrt (DLR), Oberpfaffenhofen, Germany \\ Correspondence to: E. Jäkel (e.jaekel@uni-leipzig.de)
}

Received: 14 September 2012 - Published in Atmos. Meas. Tech. Discuss.: 10 October 2012

Revised: 22 January 2013 - Accepted: 4 February 2013 - Published: 1 March 2013

\begin{abstract}
Spectral airborne upward and downward irradiance measurements are used to derive the area-averaged surface albedo. Real surfaces are not homogeneous in their reflectivity. Therefore, this work studies the effects of the heterogeneity of surface reflectivity on the area-averaged surface albedo to quantify how well aircraft measurements can resolve the small-scale variability of the local surface albedo. For that purpose spatially heterogeneous surface albedo maps were input into a 3-dimensional (3-D) Monte Carlo radiative transfer model to simulate 3-D irradiance fields. The calculated up- and downward irradiances in altitudes between $0.1 \mathrm{~km}$ and $5 \mathrm{~km}$ are used to derive the areaaveraged surface albedo using an iterative retrieval method that removes the effects due to atmospheric scattering and absorption within the layer beneath the considered level. For the case of adjacent land and sea surfaces, parametrizations are presented which quantify the horizontal distance from the coastline that is required to reduce surface heterogeneity effects on the area-averaged surface albedo to a given limit. The parametrization which is a function of altitude, aerosol optical depth, single scattering albedo, and the ratio of local land and sea albedo was applied for airborne spectral measurements. In addition, the deviation between area-averaged and local surface albedo is determined for more complex surface albedo maps. For moderate aerosol conditions (optical depth less than 0.4) and a wavelength range between 400 and $1000 \mathrm{~nm}$, the altitude and the heterogeneity of the surface albedo are the dominant factors determining the mean
\end{abstract}

deviation between local and area-averaged surface albedo. A parametrization of the mean deviation is applied to an albedo map that was derived from a Landsat image of an area in East Anglia (UK). Parametrization and direct comparison of local and area-averaged surface albedo show similar mean deviations $(20 \%$ vs. $25 \%)$ over land.

\section{Introduction}

The surface albedo $\rho$ is important with respect to the surface and atmosphere energy budgets from global to local scales. Furthermore, $\rho$ is a boundary condition for radiative transfer calculations and a key quantity for various algorithms applied in the field of atmospheric remote sensing. For example, the retrieval of microphysical parameters of optical thin clouds based on airborne or satellite-borne measurements requires representative data of the surface albedo. $\rho$ can be obtained by local measurements of the ratio of upward irradiance $F^{\uparrow}$ and downward irradiance $F^{\downarrow}$ on the surface. In the subsequent text, this surface albedo is called local surface albedo $\left(\rho_{\text {loc }}\right)$. If instead of ground-based measurements, airborne data of up- and downward irradiance at flight level are used to retrieve the surface albedo, the so-called retrieved area-averaged surface albedo $\rho_{\text {ret }}$ is obtained. This paper investigates the effects of heterogeneity in local surface albedo $\rho_{\text {loc }}$ on the retrieved area-averaged surface albedo $\rho_{\text {ret }}$. 
The effect of spatial heterogeneity in local surface albedo on up- and downward irradiances was studied by several authors (e.g. Degünther and Hänel, 2000; Li et al., 2002; Chiu et al., 2004). Degünther et al. (1998) and Degünther and Meerkötter (2000) investigated the influence of inhomogeneous snow cover on downward ultraviolet (UV) irradiance for clear-sky and a stratiform cloud situations. In both cases they concluded that the albedo heterogeneity within $40 \mathrm{~km}$ may influence the UV downward irradiance at $330 \mathrm{~nm}$ wavelength by about $2-7 \%$. For a stratiform cloud, the surface albedo heterogeneity influences the downward UV irradiance increasingly compared to the clear sky case. But at the same time the relevant area of the albedo field which has an effect on $F^{\downarrow}$ decreases due to interactions of the photons with the surface and the cloud layer, enhancing the probability of attenuation and lessening the horizontal photon spread.

The concept of effective albedo was introduced by Li et al. (2002). The effective albedo is used in 1-D radiative transfer simulations to consider for 3-D effects either due to measurements over inhomogeneous surfaces or due to 3-D radiative transfer simulations (Pirazzini and Räisänen, 2008). Chiu et al. (2004) have shown that the heterogeneity of surface albedo influences the retrieval of cloud layer properties. They applied a 3-D radiative transfer model over a checkerboard pattern of surface albedo and found that the use of an areaaveraged surface albedo instead of the heterogeneous local albedo pattern leads to underestimation of retrieved shortwave cloud absorption for partly snow-covered surfaces by up to $8 \%$. The reason for the underestimation is the nonlinear relationship (convex function) between the surface albedo and the cloud absorption.

In satellite-based remote sensing applications instead of irradiances, radiances are measured to retrieve atmospheric or surface parameters. With respect to the retrieval of the aerosol optical depth (AOD), surface albedo heterogeneity can lead to an overestimation of AOD due to the so-called adjacency effect (Lyapustin and Kaufman, 2001). This effect influences satellite or airborne radiance measurements. Adjacency increases the brightness of dark pixels and darkens bright pixels caused by horizontal photon transport. Reflected radiances of the surrounding areas, out of the sensor field of view, are scattered by the atmosphere into the field of view, thus causing a blurring effect and reducing the contrast of an image. As a result, the retrieval algorithm over dark targets overestimates the derived AOD, which causes a systematic underestimation of the retrieved surface albedo (Lyapustin, 2001). For data at high spatial resolution (25 m), the error of AOD can exceed 0.04-0.06 in the near infrared spectral range.

Ground-based radiation measurements (e.g. Bowker et al., 1985; Feister and Grewe, 1995; Aoki et al., 2002; Wuttke and Seckmeyer, 2006) deliver the local surface albedo data for point locations only, whereas satellite measurements cover larger areas (Schaaf et al., 2002). On an intermediate scale, airborne radiation measurements deliver the retrieved area-averaged surface albedo $\rho_{\text {ret }}$ (Webb et al., 2004; Wendisch et al., 2004; Coddington et al., 2008; Bierwirth et al., 2009).

Retrieving the surface albedo from airborne measurements of upward and downward irradiances involves two issues. First, atmospheric absorption and scattering due to gas molecules and aerosol particles within the layer between the flight level and the surface contribute to the airborne albedo measurement. Second, they mask the surface albedo. This masking gets larger by (i) increasing optical depth of the atmospheric layer below the flight altitude, and (ii) increasing surface albedo. To remove the influence of the atmosphere between surface and flight altitude, a nonlinear extrapolation of the albedo measured at flight level to the surface is applied (Wendisch et al., 2004). Second, the flight altitude determines the surface area surveyed by the sensor of the spectral upward irradiance $F^{\uparrow}$ (in units of $\mathrm{W} \mathrm{m}^{-2} \mathrm{~nm}^{-1}$ ). $F^{\uparrow}$ results from the radiance integrated over the lower hemisphere, and weighted by the cosine of the zenith angle $\theta$ according to the following equation:

$F^{\uparrow}=\int_{0}^{2 \pi} \int_{0}^{1} I^{\uparrow} \cdot \mu \mathrm{d} \mu \mathrm{d} \varphi$,

with $I$ representing the radiance in units of $\mathrm{W} \mathrm{m}^{-2} \mathrm{~nm}^{-1}$ $\mathrm{sr}^{-1}, \mu$ is the cosine of the zenith angle $\theta$, and $\varphi$ the azimuth angle. Due to the $\mu$-weighting, $F^{\uparrow}$ is dominated by the upward radiance near nadir direction $(\mu=1)$. For increasing altitude, reflected radiances from a larger area contribute with significant $\mu$-weighting to the upward irradiance. Combined with effects due to multiple scattering, this leads to a "smearing" (averaging) of the surface albedo as seen by the downward looking airborne irradiance instrument. Thus, the agreement between the area-averaged surface albedo $\rho_{\text {ret }}$ retrieved by the method of Wendisch et al. (2004), based on airborne measurements and the heterogenous local surface albedo $\rho_{\text {loc }}$, depends on the flight altitude and aerosol properties.

Since many remote sensing applications need knowledge about $\rho_{\text {loc }}$ instead of $\rho_{\text {ret }}$, this paper investigates how well the surface albedo can be spatially-resolved by airborne measurements depending on the heterogeneity of the local surface albedo and flight altitude. A parametrization of the mean deviation between local and retrieved area-averaged surface albedo is provided which can be used for sensitivity studies of, e.g. certain satellite retrievals. In Sect. 2, modeling and experimental tools are introduced. Section 3 analyzes the simplified case of two adjacent surface types (sea and land). Furthermore, more complex surface albedo patterns are investigated and a parametrization is provided to estimate the mean deviation between $\rho_{\text {loc }}$ and $\rho_{\text {ret }}$. A summary of the findings and conclusions are given in Sect. 4. 


\section{Methods and materials}

\subsection{Modeling}

Radiative transfer simulations are performed with the libRadtran package (Mayer and Kylling, 2005). The heterogeneity of the surface albedo is considered by using the 3-D Monte Carlo model MYSTIC (Monte Carlo code for the physically correct tracing of photons in cloudy atmospheres), which is one of the solvers available within libRadtran (Mayer, 2009). With MYSTIC the radiation field is modeled by tracing individual photons on their paths through the 3-D atmosphere. The model domain is divided into grid cells; within each grid cell the atmospheric optical properties are constant. MYSTIC applies periodic boundary conditions, which needs to be considered for the setup of the model domain. For gas absorption the LOWTRAN parametrization by Pierluissi and Peng (1985), as adapted from SBDART (Ricchiazzi and Gautier, 1998), was used. The extraterrestrial spectrum is taken from Gueymard (2004), which is averaged over $1 \mathrm{~nm}$ wavelength intervals in the solar spectral region. Profiles of pressure, temperature, density, and gases are taken from profiles given in Anderson et al. (1986) as input for the simulations. The aerosol particle properties are specified by the spectral aerosol optical depth, the single scattering albedo $\tilde{\omega}$ and the asymmetry parameter $g$. Furthermore, Lambertian surface reflection is assumed in this study.

The accuracy of the Monte Carlo results is determined by the number of traced photons. In order to balance computational efforts and accuracy, the photon number $N$ was adjusted such that a given relative standard deviation $\sigma$ of the result was achieved. For our application $\sigma$ is set to $5 \%$, which corresponds to the accuracy of the airborne irradiance measurements (Wendisch et al., 2001; Wendisch and Mayer, 2003).

\subsection{Airborne radiation measurements}

The Spectral Modular Airborne Radiation measurement sysTem (SMART)-Albedometer (Wendisch et al., 2001; Jäkel et al., 2005; Bierwirth, 2008; Ehrlich et al., 2008) was installed on a Partenavia P68B aircraft to measure upward and downward irradiances $(350-1000 \mathrm{~nm})$ with an accuracy of $5 \%$. The entrance optics of the SMART-Albedometer were leveled during the flight using a horizontal stabilization system (Wendisch et al., 2001), which assures a clear separation between photons from the upper and the lower hemispheres.

Airborne radiation measurements were collected during the INSPECTRO (INfluence of clouds on SPECtral actinic flux in the lower TROposphere) campaign in East Anglia, UK, in September 2002 (Kylling et al., 2005; Thiel et al., 2008). In particular, data measured in cloudless conditions on 12 September 2002 are used in this paper. Flights were made over sea and land surfaces (predominantly agriculture, villages, and occasional areas of woodland) in a triangular horizontal flight pattern within an area of $20 \times 20 \mathrm{~km}^{2}$ at an altitude of $z_{\text {flight }}=500 \mathrm{~m}$. The input parameters for the corresponding extrapolation method are taken from previous INSPECTRO publications (Kylling et al., 2005; Thiel et al., 2008): the AOD is approximated by using the Ångstrøm formula $\mathrm{AOD}=\beta \cdot \lambda^{-\alpha}$ and respective Ångstrøm coefficients $\alpha=1.3$, and $\beta=0.044$. This gives an $\mathrm{AOD}=0.1$ at $532 \mathrm{~nm}$ wavelength. These values were derived from the data set of a ground-based elastic backscattering lidar system (Gobbi et al., 2000), which measured within the time frame of the flights during INSPECTRO. According to Kylling et al. (2005) the single scattering albedo $\tilde{\omega}$ and asymmetry parameter $g$ of the maritime aerosol particles are set to $\tilde{\omega}=0.98$ and $g=0.75$, respectively.

The up- and downward irradiances in flight level $z_{\text {flight }}$ were used to retrieve the area-averaged surface albedo $\rho_{\text {ret }}$ (Wendisch et al., 2004). The method requires that the measured and simulated spectra of the downward irradiances in $z_{\text {flight }}$ agree within the measurement uncertainties. The iterative extrapolation algorithm starts with a given standard albedo $\rho_{0}$, which is used as a first guess of the surface albedo. The upward and downward irradiances are calculated with the 1-D radiative transfer solver DISORT (also implemented in libRadtran) for flight level $z_{\text {flight }}$ and the surface $z=0$. The ratio of the modeled albedo at the surface, $\rho_{n}(z=0)$, and at flight level, $\rho_{n}\left(z_{\text {flight }}\right)$, is used to obtain the surface albedo for the next iteration step $\rho_{n+1}$ :

$\rho_{n+1}(z=0)=\frac{\rho_{n}(z=0)}{\rho_{n}\left(z_{\text {flight }}\right)} \cdot \rho_{\text {meas }}\left(z_{\text {flight }}\right) \quad n=0,1,2, \ldots$

As the iteration proceeds, the resulting surface albedo converges to the area-averaged surface albedo. The algorithm is stopped if

$\left|1-\frac{\rho_{n+1}(z=0)}{\rho_{n}(z=0)}\right|<0.02$.

\section{2-D surface albedo heterogeneity}

\subsection{Adjacent sea and land surfaces}

\subsubsection{Synthetic surface albedo maps}

Simulated measurement flights along a horizontal path perpendicular to a straight coastline separating an area of low surface albedo (sea) and higher surface albedo (land) are used to test the impact of the flight altitude and aerosol properties on the retrieved area-averaged surface albedo $\rho_{\text {ret }}$ (see Fig. 1). $\rho_{\text {ret }}$ is influenced by both sea and land surface albedo, depending on the distance from the coastline. The position of an arbitrary point on the surface is given by Cartesian coordinates $(x, y, 0) . d$ denotes the horizontal distance between Cartesian coordinate point $(x, y, 0)$ and the foot point of the aircraft (vertical projection of aircraft position on surface). $\varphi$ describes the azimuth angle in the polar coordinate 


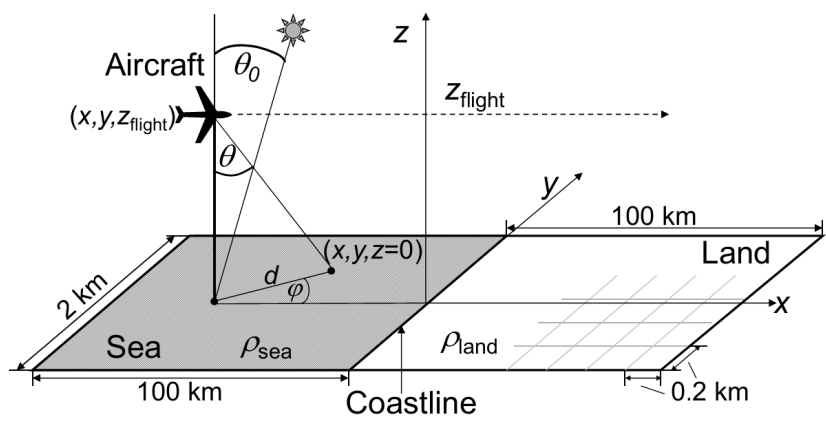

Fig. 1. Synthetic flight geometry with aircraft flying in altitude $z_{\text {flight }}$. The aircraft flies perpendicular to the coastline at constant altitude.

system, $\theta_{0}$ refers to the solar zenith angle and $\theta$ represents the zenith angle between the nadir direction and the line between the aircraft in level $z_{\text {flight }}$ and position $(x, y, 0)$. In cases where no atmospheric masking is considered, the area-averaged surface albedo retrieved from aircraft measurements $\rho_{\text {ret }}\left(x, y, z_{\text {flight }}\right)$ is a mix of the local surface albedo $\left(\rho_{\text {loc }}\right)$ of sea and land. For a Lambertian surface and neglecting atmospheric masking, the upward radiance $I^{\uparrow}(z)$ in altitudes $z \leq z_{\text {flight }}$ is isotropic, i.e.

$$
\begin{aligned}
I^{\uparrow}(x, y, z & \left.\leq z_{\text {flight }}\right)=\frac{F^{\uparrow}\left(x, y, z \leq z_{\text {flight }}\right)}{\pi} \\
& =\frac{F^{\downarrow}\left(x, y, z \leq z_{\text {flight }}\right) \cdot \rho_{\text {loc }}(x, y, 0)}{\pi}
\end{aligned}
$$

for $F^{\downarrow}\left(x, y, z \leq z_{\text {flight }}\right)=F^{\downarrow}(x, y, 0)$, the downward irradiance, $F^{\downarrow}$, is constant with altitude. Using Eq. (1) we obtain

$$
F^{\uparrow}\left(x, y, z \leq z_{\text {fight }}\right)=\int_{0}^{2 \pi} \int_{0}^{1} \frac{F^{\downarrow}(x, y, 0)}{\pi} \cdot \rho_{\mathrm{loc}}(x, y, 0) \cdot \mu \mathrm{d} \mu \mathrm{d} \varphi,
$$

with $\rho_{\mathrm{loc}}(x, y, 0)$ the local surface albedo at position $(x, y, 0)$. For the albedo at flight level, which in this case corresponds to the area-averaged surface albedo, we obtain

$$
\begin{aligned}
& \rho_{\text {ret }}\left(x, y, z_{\text {flight }}\right)=\frac{F^{\uparrow}\left(x, y, z_{\text {flight }}\right)}{F^{\downarrow}\left(x, y, z_{\text {flight }}\right)} \\
&=\frac{1}{\pi} \int_{0}^{2 \pi} \int_{0}^{1} \rho_{\text {loc }}(x, y, z=0) \cdot \mu \mathrm{d} \mu \mathrm{d} \varphi .
\end{aligned}
$$

According to this equation, $\rho_{\text {ret }}$ is a weighted mean of the local surface albedo field with the weighting function $\mu$, which can be calculated from the flight altitude and the horizontal distance $d$ by the following:

$\mu=\cos \theta=\cos \left[\arctan \frac{d(x, y)}{z}\right]$.

In cases of the assumed surface albedo scenario, we can split the integral in Eq. (6) with respect to land and sea surface albedo:

$$
\begin{aligned}
\rho_{\text {ret }}\left(x, y, z_{\text {flight }}\right) & =\frac{1}{\pi} \int_{\varphi} \int_{\mu} \rho_{\text {sea }} \cdot \mu \mathrm{d} \mu \mathrm{d} \varphi \\
& +\frac{1}{\pi} \int_{\varphi} \int_{\mu} \rho_{\text {land }} \cdot \mu \mathrm{d} \mu \mathrm{d} \varphi .
\end{aligned}
$$

Introducing the sea-land surface albedo ratio $\delta=\rho_{\text {land }} / \rho_{\text {sea }}$, Eq. (8) is expressed as follows:

$$
\begin{aligned}
\rho_{\text {ret }}\left(x, y, z_{\text {flight }}\right) & =\frac{1}{\pi} \int_{\varphi} \int_{\mu} \rho_{\text {sea }} \cdot \mu \mathrm{d} \mu \mathrm{d} \varphi \\
& +\frac{1}{\pi} \int_{\varphi} \int_{\mu} \delta \cdot \rho_{\text {sea }} \cdot \mu \mathrm{d} \mu \mathrm{d} \varphi .
\end{aligned}
$$

We define a critical distance $d_{\mathrm{c}}$ where the area-averaged surface albedo has a maximum deviation of $10 \%$ of the given local surface albedo. The threshold of $10 \%$ combines the measurement uncertainty of the SMART-Albedometer and the relative standard deviation of the photon noise. Since the local surface albedo of the sea is lower than that of land we can write

$\rho_{\text {ret }}\left(d_{\mathrm{c}}\right)=1.1 \cdot \rho_{\text {sea }}$,

and Eq. (9) simplifies to the following equation:

$$
\frac{1}{\pi} \int_{\varphi} \int_{\mu} \mu \mathrm{d} \mu \mathrm{d} \varphi+\frac{1}{\pi} \int_{\varphi} \int_{\mu} \delta \cdot \mu \mathrm{d} \mu \mathrm{d} \varphi=1.1 .
$$

If atmospheric masking is neglected, the smoothing of the upward irradiance and so the area-averaged surface albedo solely depends on the surface albedo ratio $\delta$ and not on the individual albedo values of sea and land.

In the following the atmospheric masking is introduced and the impact of aerosol properties (AOD, $\tilde{\omega}, g$ ) on the critical distance $d_{\mathrm{c}}$ is quantified using measured and simulated spectral irradiance (up- and downward) at flight altitude $z_{\text {flight }}$. Local surface albedo maps consisting of two fields which characterize sea and land are generated for a domain of $2 \times 200 \mathrm{~km}^{2}$ with a horizontal grid size of $0.2 \times 0.2 \mathrm{~km}^{2}$ (see Fig. 1). In a first step, the sea albedo is fixed to 0.026 following Bowker et al. (1985) at $\lambda=450 \mathrm{~nm}$, whereas the land albedo is varied with respect to different reflectivity properties to test various sea-land surface albedo ratios $\delta$. For some cases, also the sea albedo is adjusted. The sensitivity of $d_{\mathrm{c}}$ on the aerosol properties are tested for AOD-values between 0.0 and 0.4 , a $\tilde{\omega}$-range of 0.65 to 0.98 and asymmetry parameters between 0.65 and 0.85 . Synthetic flights are performed in different flight altitudes $z_{\text {flight }}$ between 0.2 and $2.0 \mathrm{~km}$ in $0.2 \mathrm{~km}$ increments. Upward and downward irradiances are calculated for the entire model grid. $F^{\downarrow}$ and $F^{\uparrow}$ values of all grid cells parallel to the coastline are averaged to minimize the uncertainties due to photon statistics. The averaged irradiances simulated for the different altitudes are 

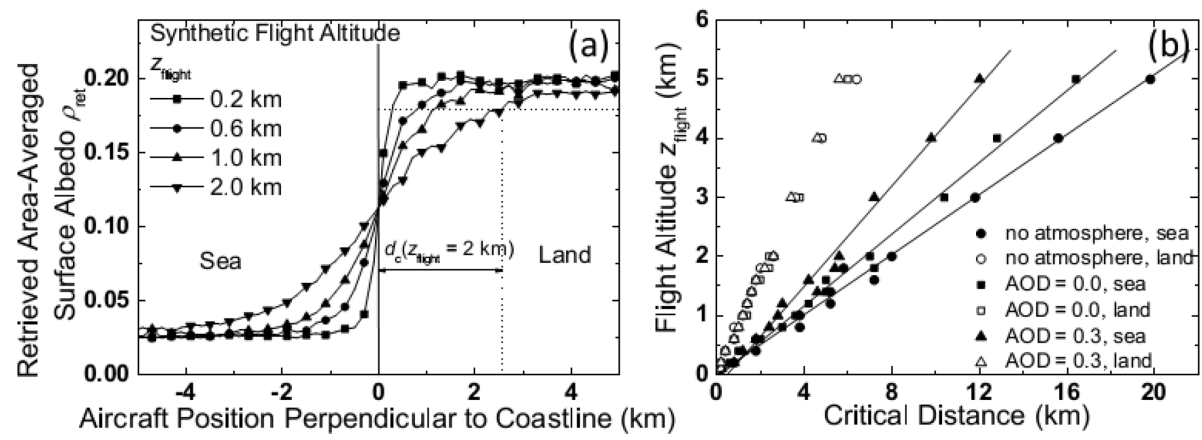

Fig. 2. (a) Cross-section of retrieved surface albedo $\rho_{\text {ret }}$ across the coastline (solar zenith angle $\theta_{0}=30^{\circ}$, aerosol optical depth $A O D=0.3$, single scattering albedo $\tilde{\omega}=0.75$, asymmetry parameter $g=0.75)$. (b) Critical distance to coastline dependent on flight altitude $\left(\theta_{0}=30^{\circ}\right.$, $\tilde{\omega}=0.75, g=0.75$ ).

used to retrieve $\rho_{\text {ret }}\left(z_{\text {flight }}\right)$ following the method described by Wendisch et al. (2004). Exemplarily, Fig. 2a shows the retrieved area-averaged surface albedos $\rho_{\text {ret }}$ at $450 \mathrm{~nm}$ that results from the assumed flight perpendicular to the coastline for different altitudes $(\mathrm{AOD}=0.3, \tilde{\omega}=0.75, g=0.75, \delta=7.7)$. With increasing distance from the coastline $(x=0, y, 0), \rho_{\text {ret }}$ converges to the local surface albedo of sea $\rho_{\text {sea }}=0.026$ and land $\rho_{\text {land }}=0.2$. When flying above sea in direction perpendicular to the coastline, the retrieved surface albedo will increase due to the impact of the brighter surface albedo of the land area. With increasing altitude the contrast of local sea and land surface albedo smoothes out. The $10 \%$-threshold criterion is indicated by the horizontal dashed line. As an example, the critical distance $d_{\mathrm{c}}$ over land for $z_{\text {flight }}=2 \mathrm{~km}$ indicated by the vertical dashed line is $2.4 \mathrm{~km}$.

Figure $2 \mathrm{~b}$ displays the critical distance as a function of the flight altitude for different values of AOD above land and above sea and for simulations without atmosphere (no Rayleigh scattering and AOD $=0$ ). For both types of surfaces, a linear dependence between $d_{\mathrm{c}}$ and $z_{\text {flight }}$ is obvious. The deviation from the linear fit between critical distance and flight altitude results from the photon noise. The higher the flight altitude the more impact there is on the adjacent surface and the aircraft needs to fly in a greater distance from the coastline to measure an undisturbed surface albedo. For greater optical depth this effect gets smaller and the critical distance decreases, which is more obvious over sea than over land. The geometry effect excluding atmospheric masking is represented by the case without atmosphere. For this specific case the maximal critical distance is derived.

For each combination of AOD, $\tilde{\omega}, g$, solar zenith angle $\theta_{0}$ $\left(30^{\circ}, 50^{\circ}\right)$, wavelength $(450 \mathrm{~nm}, 645 \mathrm{~nm})$, and surface albedo ratio investigated in the simulations a linear regression line was fitted to the slope defined by the ratio $d_{\mathrm{c}} / z_{\text {flight }}$. Figure $3 \mathrm{a}$ shows that the slope and AOD have a linear relationship, which is more pronounced for absorbing aerosols with lower single scattering albedo. In contrast, the slope is not sensitive to the AOD of highly scattering aerosols $(\tilde{\omega}=0.98)$. The slope and the surface albedo ratio reveal a nonlinear increase with increasing ratio which can be approximated by a logarithmic fit function. As expected, the higher the ratio between $\rho_{\text {land }}$ and $\rho_{\text {sea }}$, the higher $d_{\mathrm{c}}$ is, as shown for AOD $=0.0$ and $\mathrm{AOD}=0.4$ in Fig. $3 \mathrm{a}$ for $\tilde{\omega}=0.75$. Slope calculations for different solar zenith angles and wavelengths exhibit no distinct dependence of the slopes on these two parameters. Also, the effect of asymmetry parameter is negligible within the uncertainties of the linear regression of the slopes.

As a result a parametrization is provided that allows the calculation of the slope $d_{\mathrm{c}} / z_{\text {flight }}$ and the determination of the relation between flight altitude and critical distance from the coastline. Several multiple regressions were performed with different combinations of dependent variables (e.g. $\theta_{0}$, $\lambda, \mathrm{AOD}, \tilde{\omega}, \delta, \ln \delta$, and $\left.\rho_{\text {sea }}\right)$. The correlation coefficients of the parameterizations were within a range of 0.62 to 0.98 . Finally, just the parameters AOD, $\tilde{\omega}$, and $\delta$ and their combination were chosen for the parametrization, which has the following form:

$$
\frac{d_{\mathrm{c}}}{z_{\text {flight }}}=a_{0}+a_{1} \cdot \ln \delta+a_{2} \cdot \mathrm{AOD}+a_{3} \cdot \frac{\mathrm{AOD}}{\delta}+a_{4} \cdot \tilde{\omega} \cdot \delta,
$$

with $a_{0}=0.162 \pm 0.079, \quad a_{1}=1.401 \pm 0.049, \quad a_{2}=-2.771$ $\pm 0.135, a_{3}=6.526 \pm 0.721$, and $a_{4}=0.082 \pm 0.004$. This parametrization shows a correlation coefficient of 0.98 . The parameterized slopes and the slopes derived from the radiative transfer simulations are shown in Fig. 3b. The error bars are given for the slope of the simulations results from their fit uncertainty, whereas the error bars of the parameterized slopes include the uncertainty of the coefficients derived from the multiple regression. Additionally, the one-to-one line is shown in Fig. 3b. The validity of this parametrization was tested for wavelengths where extinction is mainly caused by aerosols. For wavelength regions with strong molecular absorption or scattering, Eq. (12) should not be applied. Therefore, the parametrization is restricted to a wavelength range between 400 and $1000 \mathrm{~nm}$

So far the parametrization was considered for surfaces with a lower albedo than the albedo of the adjacent surface $(\delta>1)$ and a surface albedo ratio $\delta$ defined as the ratio 

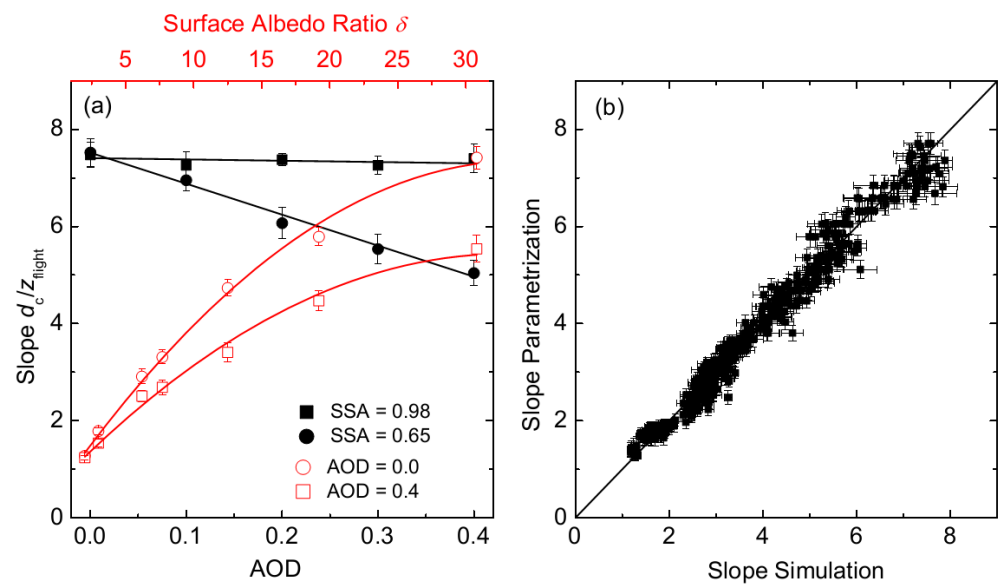

Fig. 3. (a) Slope $d_{\mathrm{c}} / z_{\text {flight }}$ as a function of aerosol optical depth $\left(\theta_{0}=30^{\circ}, \delta=30.8, g=0.75, \tilde{\omega}=0.65\right.$ and 0.98 ), and surface albedo ratio $\delta=\rho_{\text {land }} / \rho_{\text {sea }}\left(\theta_{0}=30^{\circ}, \tilde{\omega}=0.75, g=0.65, \mathrm{AOD}=0.0\right.$ and 0.4$)$. (b) Relationship of the slopes derived from the radiative transfer simulation and the parameterized slopes. Additionally, the one-to-one line is plotted.
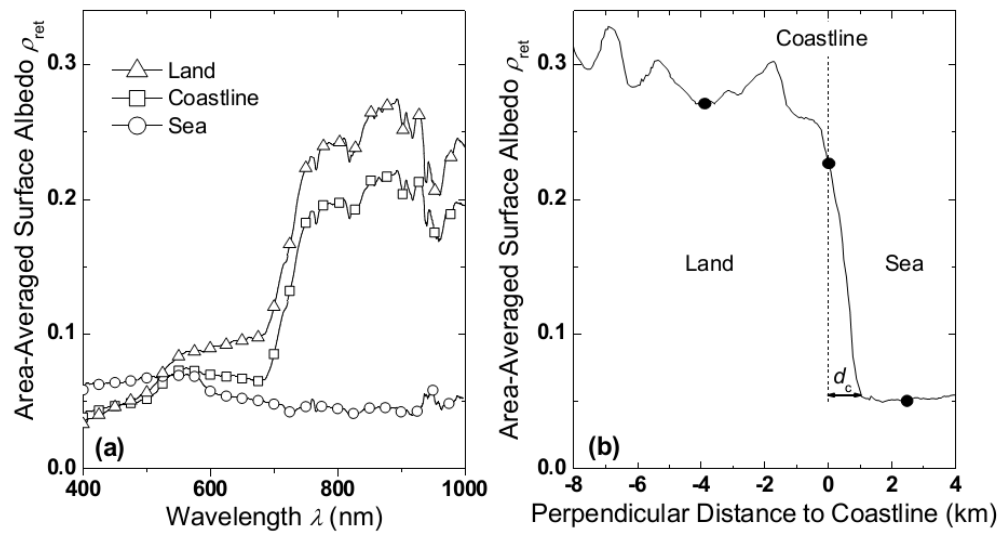

Fig. 4. (a) Measured spectra of area-averaged surface albedo $\rho_{\text {ret }}$ for land, sea, and coastline. (b) $\rho_{\text {ret }}$ for 870 nm wavelength along the coastal overflight. The black dots mark the measurement points of the spectra from (a).

between land and sea albedo. To generalize, $\delta$ is now defined as follows:

$\delta=\rho_{1} / \rho_{2}$,

with $\rho_{1}$ the surface albedo of the adjacent area and $\rho_{2}$ the surface albedo of the area which is overflown. Exemplarily, to derive the critical distance over land, $\delta$ is calculated by $\delta=\rho_{\text {sea }} / \rho_{\text {land }}$. Out of it a separate parametrization is derived for $0<\delta<2$. Since the aerosol has low impact for this $\delta$ range on the slope (cf. Fig. $2 \mathrm{~b}$ over land), the parametrization is given by the following:

$$
\frac{d_{\mathrm{c}}}{z_{\text {flight }}}=|(-1.448 \pm 0.018)+(1.334 \pm 0.0334) \cdot \delta| .
$$

Note that the right-hand side of Eq. (14) needs to be written in absolute value bars, otherwise the slope would be negative when $\rho_{1}<\rho_{2}$.

\subsubsection{Realistic surface albedo maps derived from INSPECTRO data}

The parametrization given by Eq. (12) is applied to airborne surface albedo measurements performed during the INSPECTRO campaign (Thiel et al., 2008). The example flight track contained an overpass over a mixture of small settlements and agriculture land with variable vegetation and the North Sea. Typical spectra of the area-averaged surface albedo retrieved from measurements in $500 \mathrm{~m}$ altitude are presented in Fig. 4a. The spectral vegetation step near $700 \mathrm{~nm}$ wavelength is clearly identified. Also, the increase of the surface albedo over land and near the coastline is obvious. To estimate the critical distance from the coastline $d_{\mathrm{c}}$, the highest surface albedo ratio within the measured spectral range is needed. For this example we found the maximum value of $\delta=3.8$ at $\lambda=870 \mathrm{~nm}$ with $\rho_{\text {sea }}=0.06$ and $\rho_{\text {land }}=0.23$ taken from Bowker et al. (1985). Figure 4b shows the retrieved area-averaged surface albedo for $870 \mathrm{~nm}$ wavelength along 

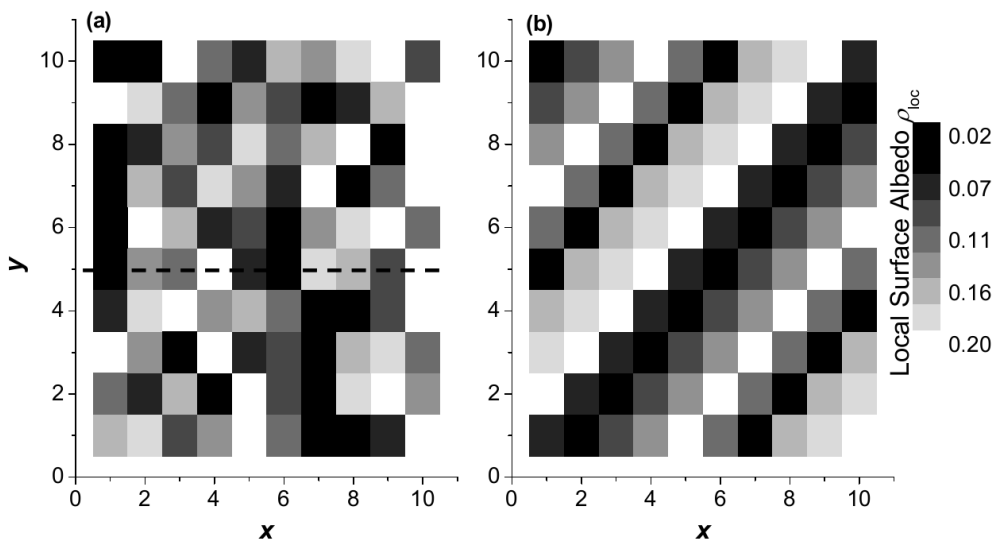

Fig. 5. Albedo maps with different distributions: (a) randomly and (b) systematically with variable grid size between 0.1 and $2 \mathrm{~km}$. The dashed line marks the position of a cross-section through the model domain.
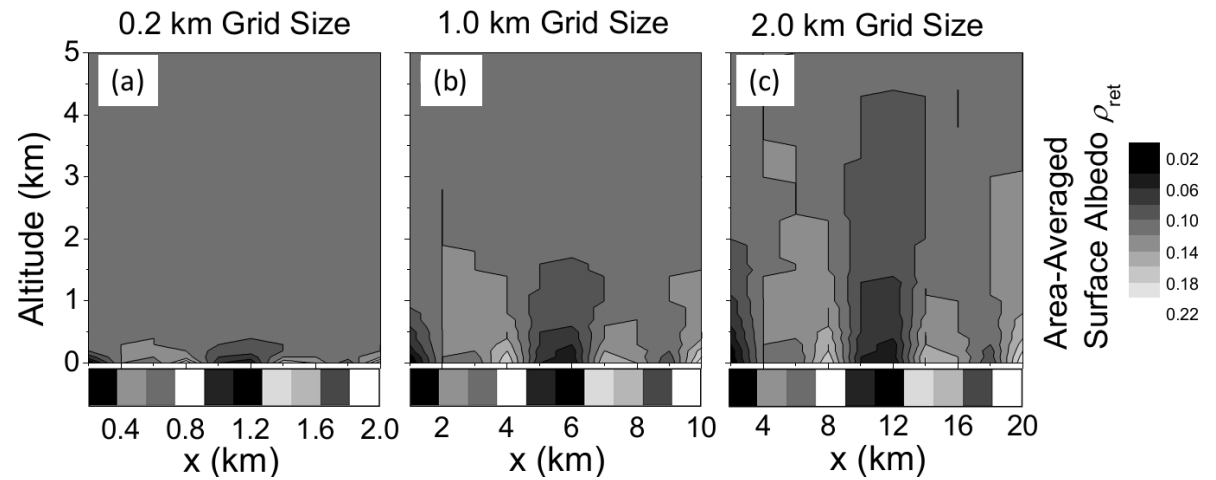

Fig. 6. Vertical cross-section of the retrieved area-averaged surface albedo $\rho_{\text {ret }}$ for (a) 0.2 , (b) $1 \mathrm{~km}$, and (c) $2 \mathrm{~km}$ resolution at $450 \mathrm{~nm}$ wavelength.

the flight track crossing the coastline. From this figure we can derive a critical distance of $1.0 \mathrm{~km}$. The parametrization after Eq. (12) with $\mathrm{AOD}=0.044$ at $870 \mathrm{~nm}, \tilde{\omega}=0.98$, and $\delta=3.8$ gives a slope of $d_{\mathrm{c}} / z_{\text {flight }}=2.29$ that corresponds to a critical distance $d_{\mathrm{c}} \approx 1.1 \mathrm{~km}$.

\subsection{Inhomogeneous albedo maps}

\subsubsection{Synthetic albedo maps}

The deviation between retrieved area-averaged surface albedo $\rho_{\text {ret }}$ and local surface albedo $\rho_{\text {loc }}$ is presented for a more complex situation of an inhomogeneous surface. A set of parameters is varied $\left(\theta_{0}, g, \tilde{\omega}, \mathrm{AOD}, \lambda\right.$ and grid size of each homogeneous surface pixel). The procedure is similar to that applied in Sect. 3.1. However, for this complex surface albedo map the relative deviation $\Delta$ of each surface pixel is the following:

$\Delta=\frac{\rho_{\text {ret }}-\rho_{\text {loc }}}{\rho_{\text {loc }}} \cdot 100 \%$, where $\rho_{\text {loc }}$ and $\rho_{\text {ret }}$ are calculated and averaged over the entire model domain. For an estimation of this mean deviation a parametrization will be given.

In a first step, synthetic albedo maps of $10 \times 10$ grid cells are generated, where the model domain size is varied between 1 and $20 \mathrm{~km}$. Local surface albedo values ranging between 0.02 and 0.2 (appropriate range for surfaces like water, grassland, sand and concrete) are distributed over the $10 \times 10$ grid with variable grid cell size $s$. An example is shown in Fig. 5. The albedo values in both plots have the same statistics but are distributed differently. In Fig. 5a, the ten albedo values are randomly distributed, whereas the distribution in Fig. $5 \mathrm{~b}$ is systematical with a shift of the albedo distribution by one pixel from one horizontal grid line to the following line.

MYSTIC simulations of upward and downward irradiances were performed for a set of input parameters. Figure 6 shows the vertical distribution of the retrieved $\rho_{\text {ret }}$ at different altitudes $z_{\text {flight }}$ along a cross-section through the model domain at all grid cells along $y=5$ as illustrated in Fig. 5a (for $\theta_{0}=50^{\circ}, \mathrm{AOD}=0.3, g=0.65, \tilde{\omega}=0.75$ ) up to $5 \mathrm{~km}$ altitude. For the most heterogeneous surface albedo 

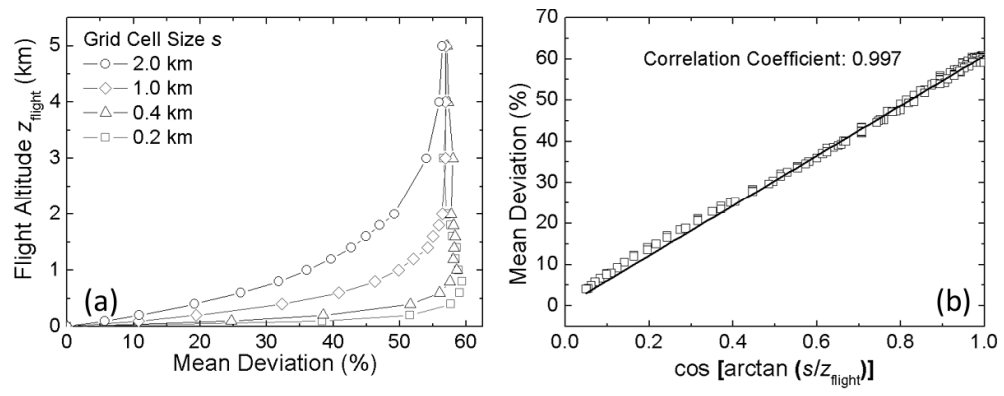

Fig. 7. (a) Vertical profile of the mean albedo deviation $\bar{\Delta}$ for different horizontal resolutions. (b) Correlation of mean albedo deviation and $\cos \theta$ with $\theta=\arctan \left(s / z_{\text {flight }}\right)$.

field (Fig. 6a) with $0.2 \mathrm{~km}$ resolution, the horizontal variability of $\rho_{\text {ret }}$ can be already neglected at $0.5 \mathrm{~km}$ altitude (relative standard deviation is less than $5 \%$ ). Increasing the resolution to $1 \mathrm{~km}$ (Fig. 6b) and $2 \mathrm{~km}$ (Fig. 6c) significantly increases the level where the horizontal variability of $\rho_{\text {ret }}$ can be neglected ( 3.0 and $4.5 \mathrm{~km}$, respectively).

In a next step, the mean deviation $\bar{\Delta}$ of the entire model domain is calculated, which is shown as a function of altitude in Fig. 7a (for $\theta_{0}=50^{\circ}, \mathrm{AOD}=0.3, g=0.65, \tilde{\omega}=0.75$ ). The nonlinear increase of $\bar{\Delta}$ is strongly affected by the grid cell size $s$ of the local albedo fields. For high altitudes the mean deviation converges for all grid cell sizes likewise to one value. In this altitude the area-averaged surface albedo $\rho_{\text {ret }}$ corresponds to the mean local surface albedo $\bar{\rho}_{\text {loc }}$ of the entire model domain. Consequently, the maximum value $\bar{\Delta}_{\max }$ corresponds to the mean deviation between $\bar{\rho}_{\text {loc }}$ and $\rho_{\text {loc }}$ :

$\bar{\Delta}_{\max }=$ mean $\left(\frac{\bar{\rho}_{\text {loc }}-\rho_{\text {loc }}}{\rho_{\text {loc }}}\right)$.

Both parameters, $s$ and $z_{\text {flight }}$, can be combined by the zenith angle $\theta=\arctan \left(s / z_{\text {flight }}\right)$. As shown in Fig. 7b, a linear regression between $\cos \theta$ and the mean deviation $\bar{\Delta}$ of the form,

$\bar{\Delta}=a \cdot \cos \theta=a \cdot \cos \left(\arctan \frac{s}{z_{\text {flight }}}\right)$,

can be fitted to the data points. Exemplarily, the slopes of all studied cases range between 59 and 61 with a correlation coefficient of 0.99 which reveals no significant differences for $a$. The impact of AOD and $\theta_{0}$ was tested by comparing calculations for AOD values ranging between 0.1 and 0.3 , and solar zenith angles of 30 and $50^{\circ}$. No significant difference was found, so that for complex local surface albedo patterns (assuming moderate AOD and a wavelength range of 400$1000 \mathrm{~nm}$ ), scattering processes within the atmosphere can be neglected compared to surface albedo inhomogeneity effects.

Table 1 summarizes the fit results as function of the surface albedo ranges. As expected, expanding the albedo range leads to an increase of $\bar{\Delta}$. Arctic situations with alternating bright (snow covered) and dark regions (open sea) would
Table 1. Fit results of Eq. (17).

\begin{tabular}{lccc}
\hline $\begin{array}{l}\text { albedo range } \\
\left(\rho_{\min } / \rho_{\max }\right)\end{array}$ & $a$ (uniform) & $a$ (random) & $\begin{array}{c}\text { mean theoretical } \\
\text { convergence } \\
\text { value } \bar{\Delta}_{\max }\end{array}$ \\
\hline 0.028 & 205 & 220 & 204 \\
0.04 & 144 & 156 & 145 \\
0.06 & 97 & 106 & 100 \\
0.08 & 72 & 81 & 76 \\
0.1 & 60 & 65 & 61 \\
0.14 & 40 & 45 & 42 \\
\hline
\end{tabular}

give the largest values for $\bar{\Delta}$. For the uniform albedo distribution, the relation between $\bar{\Delta}$ and $\cos \left[\arctan \left(s / z_{\text {flight }}\right)\right]$ is almost linear (correlation coefficient $\approx 0.99$ ), whereas tests with other albedo fields, such as the "random" distribution, have shown correlation coefficients of 0.9. The slopes of the "uniform" distributions are about $10 \%$ smaller than the slopes of the "random" distribution. Compared to the randomly distributed surface albedo case (Fig. 5a), the diagonal pattern in the albedo map of the "uniform" distribution (Fig. 5b) results in compact darker and brighter areas, which increases the altitude where the horizontal variation of the retrieved are-averaged surface albedo is negligible. Table 1 also includes the expected theoretical convergence value $\bar{\Delta}_{\max }$ which was calculated for all albedo maps using Eq. (16). Comparing these values to the slopes $a$ of the linear regression reveals a good agreement (maximum deviation $11 \%$ ). Consequently, the slope $a$ in Eq. (17) can be approximated by $\bar{\Delta}_{\text {max }}$. This becomes reasonable when, for altitudes $z$ much larger than the pixel size $s$, the cosine of $\theta=\arctan \left(s / z_{\text {flight }}\right)$ approaches unity in Eq. (17) and $\bar{\Delta}=\bar{\Delta}_{\max }=a$. To apply Eq. (17), a first guess of the albedo distribution taken from satellite data is needed to calculate $\bar{\Delta}_{\max }$.

\subsubsection{Experimental albedo maps derived from INSPECTRO}

The estimation of $\bar{\Delta}$ using Eq. (17) is applied to an experimental albedo map measured along a flight path during the 

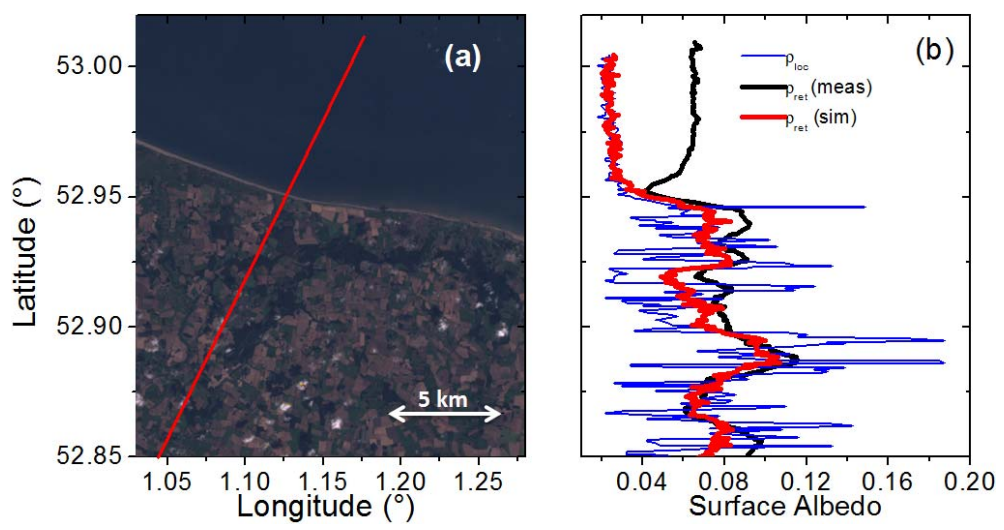

Fig. 8. (a) Landsat image and flight track of the studied area over East Anglia; (b) retrieved area-averaged surface albedo from simulations and local surface albedo taken from Landsat at $660 \mathrm{~nm}$ along the flight leg. Additionally, the retrieved area-averaged surface albedo from aircraft measurements is shown.

INSPECTRO campaign. The 2-D local surface albedo map is approximated by the surface reflectance product of the Landsat satellite. The image of the measurement area was taken in August 2007 (Global Land Cover Facility (GLCF), Goddard Space Flight Center (GSFC), 2008). It is assumed that the vegetation state of the land used for agricultural purposes is similar to the time when the aircraft measurements were performed. Each pixel of the image corresponds to a grid cell point of the surface albedo map with resolution of about $30 \mathrm{~m}$. The image in Fig. 8a shows the overall albedo map covering an area of $17 \times 19 \mathrm{~km}^{2}$. The flight track is indicated by a red line. The comparison of the reflectance values of the spectral Landsat bands over land has shown the highest contrast for band $3(0.63-0.69 \mu \mathrm{m})$. Based on that, $F^{\uparrow}$ and $F^{\downarrow}$ at $500 \mathrm{~m}$ altitude which correspond to the flight altitude were calculated for a wavelength of $660 \mathrm{~nm}$. The backward option is used for the MYSTIC simulations, which allows specifically the calculation of the observation along the flight track. Five model runs with 10000 photons each were averaged along the flight path with 280 grid cells within the albedo map of $577 \times 646$ pixels. Further input to the model is similar to that applied in Sect. 3.1. Along the flight track the area-averaged surface albedo is retrieved from simulated irradiances, as presented in Fig. 8b. The local surface albedo taken from the Landsat data at $660 \mathrm{~nm}$ wavelength is indicated by the blue line, which shows the strong spatial fluctuations of grassland/forest and wheat fields over land. Additionally, Fig. 8b displays $\rho_{\text {ret }}$ (meas) retrieved from the airborne irradiance measurements (black line), which shows reasonable agreement with $\rho_{\text {ret }}(\operatorname{sim})$ retrieved from the simulated irradiances. Over sea $\rho_{\text {ret }}(\mathrm{sim})$ is lower $(0.02$ vs. 0.07) than the results of the aircraft measurements, which might be caused by whitecaps occurring during the flight. However, it can be clearly seen that the deviation between $\rho_{\text {ret }}(\operatorname{sim})$ and $\rho_{\text {loc }}$ can locally exceed $100 \%$. The mean deviation $\bar{\Delta}$ along this flight track is calculated by averaging all $\Delta$ values which are derived from Eq. (15). The mean deviation
$\bar{\Delta}$ is about $+20 \%$. The overestimation about the dark surfaces is not completely compensated by the underestimation of the brighter surface albedos. From the parametrization given in Eq. (17), $\bar{\Delta}$ can be also estimated with the following parameters: $s=600 \mathrm{~m}$ (typical size of one homogeneous surface area); $z_{\text {flight }}=500 \mathrm{~m}$; and the slope parameter $a$, which is taken from the theoretical convergence value $\bar{\Delta}_{\max }$; cf. Eq. (16). For the entire albedo map, $\bar{\Delta}_{\max }=+45 \%$, which gives a mean deviation $\bar{\Delta}$ of $+25 \%$. Along the flight track over land, $\bar{\Delta}_{\max }$ and $\bar{\Delta}$ were calculated with +30 and $+20 \%$, respectively. The latter is lower than the value derived from the retrieval of the area-averaged surface albedo $(25 \%)$. The deviation might be caused by the uncertainty of the grid size parameter $s$ that was estimated for the albedo map.

\section{Conclusions}

This study investigates the effect of local surface albedo heterogeneity and aerosol parameters on the retrieved areaaveraged surface albedo from airborne upward and downward irradiance measurements. 3-D radiative transfer simulations were performed for different local surface albedo and aerosol situations. Calculated upward and downward irradiances in altitudes $z_{\text {flight }}$ between 0.1 and $5 \mathrm{~km}$ were used to retrieve the surface albedo using an iterative method that is based on 1-D simulations. This method is crucial to correct for atmospheric masking effects inherent in airborne measurements. The deviation of retrieved area-averaged and local surface albedo (input data of the 3-D simulations) was determined as a function of altitude, heterogeneity of the local surface albedo (spatial scale and contrasts of surface albedo), solar zenith angle $\theta_{0}$, aerosol optical depth, and single scattering albedo.

For adjacent land and sea, a critical distance $d_{\mathrm{c}}$ was defined where the retrieved area-averaged surface albedo has a 
maximal deviation of $10 \%$ of the given local surface albedo. Two simple parametrizations were derived which provide the critical perpendicular distance $d_{\mathrm{c}}$ from the coastline. For surface albedo ratios $\delta \geq 2$, the parametrization is a function of flight altitude $z_{\text {flight }}$, AOD, $\tilde{\omega}$ and $\delta$. In contrast, when $\delta<2$ the impact of the aerosol is negligible; therefore a second parametrization was provided which is only a function of the surface albedo ratio $\delta$ and flight altitude $z_{\text {flight }}$. The parametrizations include a first guess of the sea and the land albedo estimated from example values presented by Bowker et al. (1985). The critical distance $d_{\mathrm{c}}$ gets larger for albedo fields with higher surface albedo ratios. The parametrization was applied for airborne spectral measurements at $870 \mathrm{~nm}$ wavelength. The example flight pattern included one flight leg nearly perpendicular to the coastline in $500 \mathrm{~m}$ altitude. Using literature data for land and sea albedo, a critical distance of about $1.1 \mathrm{~km}$ was calculated with the parametrization for flights above sea, which could be also derived from the aircraft measurements.

For a more complex mixture of surface albedos, the mean deviation $\bar{\Delta}$ between area-averaged and local surface albedo was determined. $\bar{\Delta}$ shows a strong dependence from the altitude and the grid cell size of the homogeneous albedo areas, whereas the effect of AOD and $\theta_{0}$ can be neglected. For moderate aerosol conditions (AOD $<0.4$ ) the effect of the flight altitude and the effect of the range of heterogeneity of the local surface albedo are the dominant factors determining this deviation. To estimate $\bar{\Delta}$ of the complete area, a linear parametrization that includes the flight altitude, grid cell size of homogeneous surfaces and a slope parameter was derived. It was found that the slope parameter corresponds to the mean deviation between the local surface albedo and its areal mean value. For the calculation of the maximum deviation one can use a rough estimation of the surface albedo distribution and the range of potential albedos (e.g. $2 / 3$ water and $1 / 3$ lawn with albedo 0.06 and 0.23 , respectively), which can be derived from satellite images or land use data. Depending on the distribution of the local albedo values in the surface albedo map, correlation coefficients between 0.9 and 0.99 were derived for the parametrization. This parametrization was applied for an experimental surface albedo map. Along a flight track the surface albedo was retrieved from measured and simulated irradiances. The albedo map for the 3D simulation was derived from the surface Landsat product. Both parametrization and direct comparison of local and retrieved area-average surface albedo have shown similar mean deviations (20 and $25 \%$ ) over land. The surface albedo derived from the aircraft measurements in general follows the simulated results, which means that the local surface albedo may deviate also about $18 \%$ from the area-averaged surface albedo in average and locally up to $50 \%$.

To conclude, for flight altitudes larger or equal to the range of the size of areas with homogeneous local surface $\rho_{\text {loc }}$, the retrieved area-averaged surface albedo $\rho_{\text {ret }}$ deviates significantly from the corresponding local surface albedo. In these cases, using $\rho_{\text {ret }}$ as the boundary for remote sensing applications can lead to large uncertainties. In contrast, radiation budget and layer properties can be computed with the retrieved area-averaged surface albedo as long as $\rho_{\text {ret }}$ is representable for the considered altitudes.

Acknowledgements. This research was funded by the German Research Foundation (DFG, JA2023/2-1). We would like to thank the reviewers whose comments have helped improve this manuscript.

Edited by: S. Malinowski

\section{References}

Anderson, G., Clough, S., Kneizys, F., Chetwynd, J., and Shettle, E.: AFGL Atmospheric Constituent Profiles $(0-120 \mathrm{~km})$, Tech. Rep. AFGL-TR-86-0110, AFGL (OPI), Hanscom AFB, MA 01736, 1986.

Aoki, T., Mikami, M., and Liu, W.: Spectral albedos of desert surfaces and size distributions of soil particles measured around Qira and Aksu in the Taklimakan Desert, J. Arid Land Stud., 11, 259-266, 2002.

Bierwirth, E.: Airborne measurements of the spectral surface albedo over Morocco and its influence on the radiative forcing of Saharan dust, Ph.D. thesis, Johannes Gutenberg University, Mainz, Germany, 2008.

Bierwirth, E., Wendisch, M., Ehrlich, A., Heese, B., Tesche, M., Althausen, D., Schladitz, A., Müller, D., Otto, S., Trautmann, T., Dinter, T., von Hoyningen-Huene, W., and Kahn, R.: Spectral surface albedo over Morocco and its impact on the radiative forcing of Saharan dust, Tellus, 61, 252-269, 2009.

Bowker, D., Davis, R., Myrick, D., Stacy, K., and Jones, W.: Spectral Reflectances of Natural Targets for Use in Remote Sensing Studies, NASA RP-1139, NASA Langley Research Center, Hampton, USA, 1985.

Chiu, J., Marshak, A., and Wiscombe, W.: The effect of surface heterogeneity on cloud absorption estimates, Geophys. Res. Lett. 31, L15105, doi:10.1029/2004GL020104, 2004.

Coddington, O., Schmidt, K. S., Pilewskie, P., Gore, W. J., Bergstrom, R., Roman, M., Redemann, J., Russell, P. B., Liu, J., and Schaaf, C. C.: Aircraft measurements of spectral surface albedo and its consistency with ground-based and space-borne observations, J. Geophys. Res., 113, D17209, doi:10.1029/2008JD010089, 2008.

Degünther, M. and Hänel, G.: Simultaneous inverse determination of all optical properties of atmospheric particles: improved result by using a realistic phase function approximation, J. Atmos. Sci., 57, 2872-2882, 2000.

Degünther, M. and Meerkötter, R.: Influence of inhomogeneous surface albedo on UV irradiance: Effect of a stratus cloud, J. Geophys. Res., 105, 22755-22761, 2000.

Degünther, M., Meerkötter, R., Albold, A., and Seckmeyer, G.: Case study of the influence of inhomogeneous surface albedo on UV irradiance, Geophys. Res. Lett., 25, 3587-3590, 1998. 
Ehrlich, A., Bierwirth, E., Wendisch, M., Gayet, J.-F., Mioche, G., Lampert, A., and Heintzenberg, J.: Cloud phase identification of Arctic boundary-layer clouds from airborne spectral reflection measurements: test of three approaches, Atmos. Chem. Phys., 8, 7493-7505, doi:10.5194/acp-8-7493-2008, 2008.

Feister, U. and Grewe, R.: Spectral albedo measurements in the UV and visible region over different types of surfaces, Photochem. Photobiol., 62, 736-744, 1995.

Global Land Cover Facility (GLCF), Goddard Space Flight Center (GSFC): Landsat Surface Reflectance, Landsat TM \& ETM+, Global Land Cover Facility University of Maryland, College Park, 2008.

Gobbi, G. P., Barnaba, F., Giorgi, R., and Santacasa, A.: Altituderesolved properties of a Saharan dust event over the Mediterranean, Atmos. Environ., 34, 5119-5127, 2000.

Gueymard, C. A.: The sun's total and spectral irradiance for solar energy applications and solar radiation models, Sol. Energ., 76, 423-453, 2004.

Jäkel, E., Wendisch, M., Kniffka, A., and Trautmann, T.: Airborne system for fast measurements of upwelling and downwelling spectral actinic flux densities, Appl. Optics, 44, 434-444, 2005.

Kylling, A., Webb, A. R., Kift, R., Gobbi, G. P., Ammannato, L., Barnaba, F., Bais, A., Kazadzis, S., Wendisch, M., Jäkel, E., Schmidt, S., Kniffka, A., Thiel, S., Junkermann, W., Blumthaler, M., Silbernagl, R., Schallhart, B., Schmitt, R., Kjeldstad, B., Thorseth, T. M., Scheirer, R., and Mayer, B.: Spectral actinic flux in the lower troposphere: measurement and 1-D simulations for cloudless, broken cloud and overcast situations, Atmos. Chem. Phys., 5, 1975-1997, doi:10.5194/acp-5-1975-2005, 2005.

Li, Z., Cribb, M., and Trishchenko, A.: Impact of surface inhomogeneity on solar radiative transfer under overcast conditions, J. Geophys. Res., 107, 4294, doi:10.1029/2001JD000976, 2002.

Lyapustin, A.: Three-dimensional effects in the remote sensing of surface albedo, IEEE T. Geosci. Remote, 39, 254-263, 2001.

Lyapustin, A. and Kaufman, Y.: Role of adjacency effect in the remote sensing of aerosol, J. Geophys. Res., 106, 11909-11916, 2001.

Mayer, B.: Radiative transfer in the cloudy atmosphere, Eur. Phys. J Conf., 1, 75-99, 2009.

Mayer, B. and Kylling, A.: Technical note: The libRadtran software package for radiative transfer calculations - description and examples of use, Atmos. Chem. Phys., 5, 1855-1877, doi:10.5194/acp-5-1855-2005, 2005.

Pierluissi, J. and Peng, G.-S.: New molecular transmission band models for LOWTRAN, Opt. Eng., 24, 541-547, 1985.
Pirazzini, R. and Räisänen, P.: A method to account for surface albedo heterogeneity in single-column radiative transfer calculations under overcast conditions, J. Geophys. Res., 113, D20108, doi:10.1029/2008JD009815, 2008.

Ricchiazzi, P. and Gautier, C.: Investigation of the effect of surface heterogeneity and topography on the radiation environment of Palmer Station, Antarctica, with a hybrid 3-D radiative transfer model, J. Geophys. Res., 103, 6161-6178, 1998.

Schaaf, C. B., Gao, F., Strahler, A. H., Lucht, W., Li, X. W., Tsang, T., Strugnell, N. C., Zhang, X. Y., Jin, Y. F., Muller, J. P., Lewis, P., Barnsley, M., Hobson, P., Disney, M., Roberts, G., Dunderdale, M., Doll, C., d'Entremont, R. P., Hu, B. X., Liang, S. L., Privette, J. L., and Roy, D.: First operational BRDF, albedo nadir reflectance products from MODIS, Remote Sens. Environ., 83, 135-148, 2002.

Thiel, S., Ammannato, L., Bais, A., Bandy, B., Blumthaler, M., Bohn, B., Engelsen, O., Gobbi, G. P., Gröbner, J., Jäkel, E., Junkermann, W., Kazadzis, S., Kift, R., Kjeldstad, B., Kouremeti, N., Kylling, A., Mayer, B., Monks, P. S., Reeves, C. E., Schallhart, B., Scheirer, R., Schmidt, S., Schmitt, R., Schreder, J., Silbernagl, R., Topaloglou, C., Thorseth, T. M., Webb, A. R., Wendisch, M., and Werle, P.: Influence of clouds on the spectral actinic flux density in the lower troposphere (INSPECTRO): overview of the field campaigns, Atmos. Chem. Phys., 8, 17891812, doi:10.5194/acp-8-1789-2008, 2008.

Webb, A. R., Kylling, A., Wendisch, M., and Jäkel, E.: Airborne measurements of ground and cloud spectral albedos under low aerosol loads, J. Geophys. Res., 109, D20205, doi:10.1029/2004JD004768, 2004.

Wendisch, M. and Mayer, B.: Vertical distribution of spectral solar irradiance in the cloudless sky: A case study, Geophys. Res. Lett., 30, 1183, doi:10.1029/2002GL016529, 2003.

Wendisch, M., Heintzenberg, J., and Bussemer, M.: Measurementbased aerosol forcing calculations: the influence of model complexity, Meteorol. Z., 10, 45-60, 2001.

Wendisch, M., Pilewskie, P., Jäkel, E., Schmidt, S., Pommier, J., Howard, S., Jonsson, H. H., Guan, H., Schröder, M., and Mayer, B.: Airborne measurements of areal spectral surface albedo over different sea and land surfaces, J. Geophys. Res., 109, D08203, doi:10.1029/2003JD004392, 2004.

Wuttke, S. and Seckmeyer, G.: Spectral radiance and sky luminance in Antarctica: a case study, Theor. Appl. Climatol., 85, 131-148, 2006. 